Romulo Reis Aguiar

\title{
Desenvolvimento de um dispositivo gerador de vibroimpacto
}

\author{
DISSERTAÇÃO DE MESTRADO \\ DEPARTAMENTO DE ENGENHARIA MECÂNICA \\ Programa de Pós-graduação em \\ Engenharia Mecânica
}



Pontifícia Universidade Católica $_{\text {Do Rio de Janeiro }}$

Romulo Reis Aguiar

Desenvolvimento de um dispositivo gerador de vibroimpacto

Dissertação apresentada como requisito parcial para obtenção do grau de Mestre pelo Programa de Pós-graduação em Engenharia Mecânica do Departamento de Engenharia Mecânica da PUC-Rio

Orientador: Prof. Hans Ingo Weber 


\title{
Pontifícia Universidade Católica DO RIO DE JANEIRO
}

\section{Romulo Reis Aguiar}

\section{Desenvolvimento de um dispositivo gerador de vibroimpacto}

\begin{abstract}
Dissertação apresentada como requisito parcial para obtenção do grau de Mestre pelo Programa de Pós-graduação em Engenharia Mecânica do Departamento de Engenharia Mecânica do Centro Técnico Científico da PUC-Rio. Aprovada pela Comissão Examinadora abaixo assinada.
\end{abstract}

\author{
Prof. Hans Ingo Weber, Dr.-Ing. \\ Orientador \\ Departamento de Engenharia Mecânica - PUC-Rio
}

Prof. João Carlos Ribeiro Plácido, Ph.D. CENPES - PETROBRAS

Luiz Fernando Penna Franca, D.Sc. Drilling Mechanics Group - CSIRO Petroleum

Prof. Marcelo Amorim Savi, D.Sc. Departamento de Engenharia Mecânica - COPPE-UFRJ

Prof. José Eugênio Leal Coordenador Setorial do Centro Técnico Científico - PUC-Rio 
Todos os direitos reservados. É proibida a reprodução total ou parcial do trabalho sem autorização da universidade, do autor e do orientador.

\section{Romulo Reis Aguiar}

Graduou-se em Engenharia Mecânica pela Pontifícia Universidade Católica do Rio de Janeiro, tendo dedicado 2 anos à projetos de iniciação científica (PIBIC-CNPq) nas áreas de dinâmica de rotores e vibroimpacto. Trabalhou por 3 anos na GE Aircraft Engines, especializando-se na área de desenvolvimento de novos reparos de componentes de motores aeronáuticos. Apresentou trabalho junto com seu orientador durante o Mestrado no XXVI CILAMCE. Atualmente dedica-se ao curso de Doutorado em Engenharia Mecânica na PUC-Rio.

Ficha Catalográfica

Aguiar, Romulo Reis

Desenvolvimento de um dispositivo gerador de vibroimpacto / Romulo Reis Aguiar; orientador: Hans Ingo Weber . - Rio de Janeiro: PUC, Departamento de Engenharia Mecânica, 2006.

$146 \mathrm{f}: ; 30 \mathrm{~cm}$

Dissertação (mestrado) - Pontifícia Universidade Católica do Rio de Janeiro, Departamento de Engenharia Mecânica.

Inclui referências bibliográficas.

1. Engenharia Mecânica - Teses. 2. Colunas de Perfuração. 3. Vibração Axial. 4. Taxa de Penetração (ROP). 5. Impactos. 6. Vibroimpacto. I. Weber, Hans Ingo. II. Pontifícia Universidade Católica do Rio de Janeiro. Departamento de Engenharia Mecânica. III. Título. 


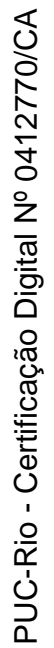

Aos meus pais, José e Marina. 


\section{Agradecimentos}

Aos meus pais, José Aguiar e Marina Reis Aguiar, que durante todo esse tempo tiveram paciência, amor e compreensão fundamentais para galgar mais este passo tão importante.

Ao professor Hans I. Weber por sua amizade, dedicação e inestimável contribuição na minha formação e elaboração deste trabalho.

À PUC-Rio, ao CNPq e à FAPERJ, pelos auxílios concedidos, sem os quais este trabalho não poderia ter sido realizado.

Aos colegas do Laboratório de Vibrações da PUC-Rio, pelas discussões e troca de idéias, fontes valiosas para o enriquecimento deste trabalho.

Ao técnico Wagner Epifânio da Cruz que, com a sua ajuda incondicional, colaborou na construção e montagem da bancada experimental.

À Denise Marques, por sua paciência e contribuição ao revisar o texto da dissertação. 


\section{Resumo}

Aguiar, Romulo Reis; Weber, Hans Ingo. Desenvolvimento de um dispositivo gerador de vibroimpacto. Rio de Janeiro, 2006. 146p. Dissertação de Mestrado - Departamento de Engenharia Mecânica, Pontifícia Universidade Católica do Rio de Janeiro.

A perfuração de rochas duras ainda é um grande desafio para as empresas de perfuração e exploração de petróleo. Uma das linhas de pesquisas atuais consiste em combinar satisfatoriamente duas técnicas para prover o aumento da taxa de penetração. Esta nova técnica vem sendo chamada de perfuração com martelo em ressonância. Esta dissertação se propõe a desenvolver o primeiro protótipo de um dispositivo que irá operar em ressonância e que será capaz de gerar forças dinâmicas expressivas. De forma resumida, este dispositivo será chamado de "RIMD" (Resonant Impact Device). Em princípio a idéia é construir um dispositivo em forma de uma "caixa preta", na qual será montada na estrutura que vibra, tendo esta caixa dois ajustes, um calibrando a freqüência de ressonância do RIMD e outro agindo sobre os impactos (folga). É conhecido de trabalhos anteriores que o tamanho da folga também possui influência sobre a freqüência natural do sistema. Desta forma, existe uma interdependência entre ambos os ajustes. Um dos primeiros passos no projeto e desenvolvimento do protótipo do RIMD é o dimensionamento do mesmo, de forma que seja pequeno o suficiente para facilitar sua construção e instrumentação no laboratório de vibrações da PUC-Rio, bem como seja representativo do sistema em tamanho real (a ser implantado na coluna de perfuração). Os componentes do RIMD envolvem um sistema massa-mola com baixo amortecimento e algum dispositivo de impacto e de variação da folga. Após a concepção e construção do protótipo, os passos seguintes do estudo são a obtenção das características do RIMD, como a faixa de freqüências o qual atua e a medição das forças impulsivas geradas. Por último, o protótipo também servirá para validar um modelo analítico que permitirá investigações posteriores neste tema, podendo gerar outras possibilidades de construção do RIMD.

\section{Palavras-chave}

colunas de perfuração, vibração axial, taxa de penetração (ROP), impactos, vibroimpacto. 


\section{Abstract}

Aguiar, Romulo Reis; Weber, Hans Ingo. Development of a vibroimpact device. Rio de Janeiro, 2006. 146p. MSc. Dissertation - Departamento de Engenharia Mecânica, Pontifícia Universidade Católica do Rio de Janeiro.

Hard rock drilling is still a great challenge for oil companies. One current line of research involves combining the two existing drilling techniques in order to enhance the rate of penetration. This new technique is called Resonance Hammer Drilling. This dissertation proposes the design and development of the first prototype that will operate in resonance, and will be capable of generating considerable dynamic forces. This device will be known as the Resonant Impact Device, or "RIMD". In principle the idea is to build some sort of "black box", which will be mounted on a vibrating structure with two switches - one calibrating the RIMD resonance frequency and the other acting on the impacts - changing the size of the gap. It is known from previous work that gap size also has influence on the system natural frequency. Therefore there is a relationship between switches. One of the first steps of RIMD design and development is device dimensioning, necessary in order to construct a scale model at the Dynamic and Vibration laboratory at PUC-Rio representative of the real size system. The real size system will be mounted on the drillstring. RIMD components involve a mass-spring system with low damping and some impact and gap variation devices. The analysis of this prototype includes obtaining key characteristics such as the range of possible frequencies and the measurement of the generated impulsive forces. Finally, the built prototype will be used to validate an analytical model that will allow further investigations on this subject providing the way to other possible constructions.

\section{Keywords}

oilwell drillstrings, axial vibration, rate of penetration (ROP), impacts, vibroimpact. 


\section{Conteúdo}

1 INTRODUÇÃO

$\begin{array}{lll}1.1 & \text { Perfuração de poços de petróleo } & 19\end{array}$

1.2 Equipamentos da sonda de perfuração 20

1.3 Colunas de perfuração 25

1.4 Brocas 28

1.5 Vibrações em colunas de perfuração 30

1.6 ROP em rochas duras e inovações no processo de perfuração 31

1.7 Objetivos do trabalho 33

1.8 Organização do trabalho 34

2 MODELAGEM DA DINÂMICA AXIAL DA COLUNA DE PERFURAÇÃO

2.1 Introdução 35

2.2 Dinâmica (Axial) da Coluna de Perfuração 37

2.3 Solução Analítica e Simulação Numérica 41

2.4 Considerações Finais 45

3 MODELAGEM DO DISPOSITIVO DE VIBROIMPACTO 46

3.1 Introdução sobre Impacto 46

3.2 Modelos Contínuos de Impacto 49

3.3 Modelagem Matemática do RIMD 54

3.4 Resultados numéricos e discussões 57

3.5 Considerações finais 67

4 METODOLOGIA E RESULTADOS EXPERIMENTAIS 69

4.1 Aparato Experimental 69

4.2 Metodologia Experimental 73

4.3 Identificação de parâmetros e Resultados Experimentais $\quad 74$

4.4 Considerações Finais 118

5 VALIDAÇÃO DO MODELO NUMÉRICO 121

5.1 Identificação dos Parâmetros do Impacto 121

5.2 Resultados Numéricos 124

5.3 Comparação Numérico-Experimental 126

5.4 Considerações Finais 137

6 CONCLUSÕES 139

6.1 Trabalhos futuros 142

$\begin{array}{ll}\text { Bibliografia } & 144\end{array}$ 


\section{Lista de Figuras}

1.1 Imagens de campo - perfuração de um poço de petróleo (site geocities.yahoo.com.br). 18

1.2 Perfuração de um poço de petróleo (site www.howstuffworks.com).

1.3 Desenho esquemático de uma plataforma de perfuração (site www.howstuffworks.com). 20

1.4 Kelly de seção reta hexagonal e quadrada [25]. 24

1.5 Comando (Drill Collar) [25]. 26

1.6 Tubo de perfuração (Drill Pipe) [25]. 26

1.7 a) Broca de diamante natural; b) broca tipo PDC

1.8 Broca tricônica com insertos

1.9 Tipos de vibração em uma coluna de perfuração [13]. 30

2.1 Tipos de vibração em uma coluna de perfuração [13]. 36

2.2 Desenho esquemático da coluna de perfuração [8]. 38

2.3 a) Broca tricônica (site geocities.yahoo.com.br/perfuracao); b) formação de três lóbulos no fundo de rochas duras, causados pelo processo de perfuração com broca tricônica [10]. 40

2.4 Espectro de amplitude dos tubos de perfuração no domínio da freqüência (em $\left.u_{T P}\left(\left(L_{T P}-L_{B H A}\right) / 2\right)\right)$. 43

2.5 Espectro de amplitude dos comandos no domínio da freqüência $\left(\mathrm{em} u_{B H A}\left(L_{B H A} / 2\right)\right)$. 43

2.6 Resposta no tempo - tubos de perfuração; $N=48.31 \mathrm{rpm}$. $\quad 44$

2.7 Resposta no tempo - comandos; $N=48.31 \mathrm{rpm}$. 44

3.1 Impacto entre dois corpos [4].

3.2 Deformação durante o impacto [4].

3.3 Fluxo de energia [4]. 49

3.4 Força de contato para o modelo de Kelvin-Voigt [15]. 51

3.5 Impacto de uma massa com uma superfície rígida para diferentes valores de velocidade: a) plano fase; b) característica força/penetração. Parâmetros utilizados: $m=2 K g k_{c}=2.1$. $10^{8} \mathrm{~N} / \mathrm{m}_{c}^{n} n_{c}=1.6 \lambda_{c}=0.6 \mathrm{~s} / \mathrm{m}$. 53

3.6 Desenho esquemático do sistema de perfuração rotativa. 54

3.7 Protótipo do RIMD. $\quad 55$

3.8 Modelagem do sistema. 56

3.9 Modelos de comparação a) 1 GDL; b) 2 GDL sem impacto. 57

3.10 Força transmitida versus freqüência de excitação a) $1 \mathrm{GDL}$;
b) 2 GDL.

3.11 Influência da distribuição de massa. Força transmitida versus freqüência. 
3.12 Influência da distribuição de massa: a) primeiro modo (detalhe);

b) segundo modo (detalhe).

3.13 Resposta em freqüência: a) velocidade; b) força de impacto. $\quad 61$

3.14 Plano fase do RIMD, $\Omega=4.53 \mathrm{~Hz}$, condição em regime. 62

3.15 Comportamento da força de impacto, $\Omega=4.53 \mathrm{~Hz}$. 63

3.16 Resposta em freqüência da força de impacto: a) aumentando $k_{2}$; b) diminuindo $k_{2}$.

3.17 Influência da rigidez do RIMD.

3.18 Condição de período- 1 . $k_{2}=90 \mathrm{~N} / \mathrm{m}$ e $\Omega=4.338 \mathrm{~Hz}$ : a) plano fase do RIMD; b) perfil da força de impacto.

3.19 Condição de período-1 transitória. $k_{2}=70 \mathrm{~N} / \mathrm{m}$ e $\Omega=$ 4.271 Hz: a) plano fase do RIMD; b) perfil da força de impacto.

3.20 Condição de período-0.5. $k_{2}=40 \mathrm{~N} / \mathrm{m}$ e $\Omega=4.196 \mathrm{~Hz}$ : a) plano fase do RIMD; b) perfil da força de impacto.

3.21 Resposta em freqüência da força de impacto: aumentando o gap. 66

3.22 Resposta em freqüência da força de impacto: diminuindo o gap. 67

4.1 Foto da bancada experimental. $\quad 70$

4.2 Desenho esquemático do experimento. $\quad 70$

4.3 Tratamento dos sinais. $\quad 73$

4.4 Correlação bancada - modelo (ver figura (4.2)). 74

4.5 Foto do experimento 1 grau de liberdade, sem impacto. $\quad 75$

4.6 Resposta em freqüência - vibração livre. $\quad 75$

4.7 Resposta no tempo: a) acelerômetro 2 (AB77); b) acelerômetro 3 (AC69).

4.8 Picos de aceleração: a) acelerômetro 2 (AB77); b) acelerômetro 3 (AC69).

4.9 Vibração livre do RIMD, distância entre acoplamentos $10 \mathrm{~cm}$ : a) resposta em freqüência; b) resposta no tempo.

4.10 Freqüências naturais, distância entre acoplamentos $10 \mathrm{~cm}$ :

a) modelo Numérico; b) experimental.

4.11 Freqüências naturais, distância entre acoplamentos $15 \mathrm{~cm}$ a) RIMD; b) sistema. 82

4.12 Resposta no tempo. Distância entre acoplamentos $15 \mathrm{~cm}$; folga $0 \mathrm{~mm} ; \Omega=3.25 \mathrm{~Hz}$. a) $F_{i}$ versus $F_{0}$; b) Acelerações.

4.13 Respostas no tempo. Distância entre acoplamentos $15 \mathrm{~cm}$; folga $0 \mathrm{~mm} ; \Omega=3.5 \mathrm{~Hz}$. a) $F_{i}$ versus $F_{0}$; b) Acelerações.

4.14 Respostas no tempo. Distância entre acoplamentos $15 \mathrm{~cm}$; folga $0 \mathrm{~mm} ; \Omega=4.5 \mathrm{~Hz}$. a) $F_{i}$ versus $F_{0}$; b) Acelerações.

4.15 Resposta no tempo. $F_{i}$ versus $F_{0}$. Distância entre acoplamentos $15 \mathrm{~cm}$; folga $0 \mathrm{~mm} ; \Omega=5.5 \mathrm{~Hz}$.

4.16 Resposta no tempo. Distância entre acoplamentos $15 \mathrm{~cm}$; folga $0 \mathrm{~mm} ; \Omega=5.5 \mathrm{~Hz}:$ a) acelerações; b) detalhe do gráfico.

4.17 Respostas no tempo. Distância entre acoplamentos $15 \mathrm{~cm}$; folga $0 \mathrm{~mm} ; \Omega=7.75 \mathrm{~Hz}$ : a) $F_{i}$ versus $F_{0}$; b) acelerações.

4.18 Respostas no tempo. Distância entre acoplamentos $15 \mathrm{~cm}$; folga $0 \mathrm{~mm} ; \Omega=9.25 H z:$ a) $F_{i}$ versus $F_{0} ;$ b) acelerações. 
4.19 Respostas no tempo. Distância entre acoplamentos $15 \mathrm{~cm}$; folga $0 \mathrm{~mm} ; \Omega=10.25 \mathrm{~Hz}$ : a) $F_{i}$ versus $F_{0}$; b) acelerações.

4.20 Respostas no tempo. Distância entre acoplamentos $15 \mathrm{~cm}$; folga $0 \mathrm{~mm} ; \Omega=11 H z$ : a) $F_{i}$ versus $F_{0}$; b) acelerações.

4.21 Respostas no tempo. Distância entre acoplamentos $15 \mathrm{~cm}$; folga $0 \mathrm{~mm} ; \Omega=12 H z:$ a) $F_{i}$ versus $F_{0}$; b) acelerações.

4.22 Respostas no tempo. Distância entre acoplamentos $15 \mathrm{~cm}$; folga $0 \mathrm{~mm} ; \Omega=13.25 \mathrm{~Hz}$ : a) $F_{i}$ versus $F_{0}$; b) acelerações.

4.23 Respostas no tempo. Distância entre acoplamentos $15 \mathrm{~cm}$; folga $0 \mathrm{~mm} ; \Omega=14.5 \mathrm{~Hz}$ : a) $F_{i}$ versus $F_{0}$; b) acelerações.

4.24 Resposta no domínio da freqüência. Distância entre acoplamentos $15 \mathrm{~cm}$; folga $0 \mathrm{~mm} ; F_{i} / F_{0}$ versus $\Omega$.

4.25 Respostas no tempo. Distância entre acoplamentos $15 \mathrm{~cm}$; folga $1 \mathrm{~mm} ; \Omega=6.25 \mathrm{~Hz}$ : a) $F_{i}$ versus $F_{0} ;$ b) acelerações.

4.26 Respostas no tempo. Distância entre acoplamentos $15 \mathrm{~cm}$; folga $1 \mathrm{~mm} ; \Omega=7 H z:$ a) $F_{i}$ versus $F_{0} ;$ b) acelerações.

4.27 Respostas no tempo. Distância entre acoplamentos $15 \mathrm{~cm}$; folga $1 \mathrm{~mm} ; \Omega=8.75 \mathrm{~Hz}:$ a) $F_{i}$ versus $F_{0} ;$ b) acelerações.

4.28 Respostas no tempo. Distância entre acoplamentos $15 \mathrm{~cm}$; folga $1 \mathrm{~mm} ; \Omega=13.5 \mathrm{~Hz}:$ a) $F_{i}$ versus $F_{0}$; b) acelerações.

4.29 Resposta no domínio da freqüência. Distância entre acoplamentos $15 \mathrm{~cm}$; folga $1 \mathrm{~mm} ; F_{i} / F_{0}$ versus $\Omega$.

4.30 Respostas no tempo. Distância entre acoplamentos $15 \mathrm{~cm}$; folga $3 \mathrm{~mm} ; \Omega=6 H z:$ a) $F_{i}$ versus $F_{0} ;$ b) acelerações.

4.31 Respostas no tempo. Distância entre acoplamentos $15 \mathrm{~cm}$; folga $3 \mathrm{~mm} ; \Omega=12.5 \mathrm{~Hz}:$ a) $F_{i}$ versus $F_{0} ;$ b) acelerações.

4.32 Resposta no domínio da freqüência. Distância entre acoplamentos $15 \mathrm{~cm}$; folga $3 \mathrm{~mm} ; F_{i} / F_{0}$ versus $\Omega$.

4.33 Resposta no domínio da freqüência. Distância entre acoplamentos $15 \mathrm{~cm}$; comparação entre folgas. $F_{i} / F_{0}$ versus $\Omega$.

4.34 Resposta no domínio da freqüência. Distância entre acoplamentos $17 \mathrm{~cm}$; comparação entre folgas. $F_{i} / F_{0}$ versus $\Omega$.

4.35 Resposta no domínio da freqüência. Distância entre acoplamentos $19 \mathrm{~cm}$; folga $0 \mathrm{~mm} ; F_{i} / F_{0}$ versus $\Omega$.

4.36 Resposta no domínio da freqüência. Distância entre acoplamentos $19 \mathrm{~cm}$; folga $1 \mathrm{~mm} ; F_{i} / F_{0}$ versus $\Omega$.

4.37 Respostas no tempo. Distância entre acoplamentos $19 \mathrm{~cm}$; folga $3 \mathrm{~mm} ; \Omega=12.5 \mathrm{~Hz}$ : a) $F_{i}$ versus $F_{0}$; b) acelerações.

4.38 Resposta no domínio da freqüência. Distância entre acoplamentos $19 \mathrm{~cm}$; folga $3 \mathrm{~mm} ; F_{i} / F_{0}$ versus $\Omega$.

101

4.39 Resposta no domínio da freqüência. Distância entre acoplamentos $19 \mathrm{~cm}$; comparação entre folgas; $F_{i} / F_{0}$ versus $\Omega$.

4.40 Resposta no tempo. $F_{i}$ versus $F_{0}$. Distância entre acoplamentos $21 \mathrm{~cm} ;$ folga $0 \mathrm{~mm} ; \Omega=2.75 \mathrm{~Hz}$.

101

4.41 Resposta no tempo. Distância entre acoplamentos $21 \mathrm{~cm}$; folga $0 \mathrm{~mm} ; \Omega=2.75 \mathrm{~Hz}$ : a) acelerações; b) detalhe do gráfico.

4.42 Respostas no tempo. Distância entre acoplamentos $21 \mathrm{~cm}$; folga $0 \mathrm{~mm} ; \Omega=5.25 \mathrm{~Hz}$ : a) $F_{i}$ versus $F_{0}$; b) acelerações. 
4.43 Respostas no tempo. Distância entre acoplamentos $21 \mathrm{~cm}$; folga $0 \mathrm{~mm} ; \Omega=6.75 \mathrm{~Hz}$ : a) $F_{i}$ versus $F_{0}$; b) acelerações.

4.44 Respostas no tempo. Distância entre acoplamentos $21 \mathrm{~cm}$; folga $0 \mathrm{~mm} ; \Omega=7.5 \mathrm{~Hz}$ : a) $F_{i}$ versus $F_{0}$; b) acelerações.

4.45 Respostas no tempo. Distância entre acoplamentos $21 \mathrm{~cm}$; folga $0 \mathrm{~mm} ; \Omega=8 H z:$ a) $F_{i}$ versus $F_{0}$; b) acelerações.

4.46 Respostas no tempo. Distância entre acoplamentos $21 \mathrm{~cm}$; folga $0 \mathrm{~mm} ; \Omega=9 H z:$ a) $F_{i}$ versus $F_{0} ;$ b) acelerações.

4.47 Respostas no tempo. Distância entre acoplamentos $21 \mathrm{~cm}$; folga $0 \mathrm{~mm} ; \Omega=12.25 \mathrm{~Hz}:$ a) $F_{i}$ versus $F_{0}$; b) acelerações.

4.48 Respostas no tempo. Distância entre acoplamentos $21 \mathrm{~cm}$; folga $0 \mathrm{~mm} ; \Omega=14.25 \mathrm{~Hz}:$ a) $F_{i}$ versus $\left.F_{0} ; \mathrm{b}\right)$ acelerações. 107

4.49 Resposta no domínio da freqüência. Distância entre acoplamentos $21 \mathrm{~cm}$; folga $0 \mathrm{~mm} ; F_{i} / F_{0}$ versus $\Omega$.

4.50 Respostas no tempo. Distância entre acoplamentos $21 \mathrm{~cm}$; folga $1 \mathrm{~mm} ; \Omega=4.5 \mathrm{~Hz}$ : a) $F_{i}$ versus $F_{0}$; b) acelerações.

4.51 Respostas no tempo. Distância entre acoplamentos $21 \mathrm{~cm}$; folga $1 \mathrm{~mm} ; \Omega=5.25 \mathrm{~Hz}:$ a) $F_{i}$ versus $F_{0} ;$ b) acelerações.

4.52 Respostas no tempo. Distância entre acoplamentos $21 \mathrm{~cm}$; folga $1 \mathrm{~mm} ; \Omega=5.75 \mathrm{~Hz}$ : a) $F_{i}$ versus $F_{0}$; b) acelerações.

4.53 Respostas no tempo. Distância entre acoplamentos $21 \mathrm{~cm}$; folga $1 \mathrm{~mm} ; \Omega=8.75 \mathrm{~Hz}$ : a) $F_{i}$ versus $F_{0}$; b) acelerações.

4.54 Respostas no tempo. Distância entre acoplamentos $21 \mathrm{~cm}$; folga $1 \mathrm{~mm} ; \Omega=11 \mathrm{~Hz}$ : a) $F_{i}$ versus $F_{0}$; b) acelerações.

4.55 Respostas no tempo. Distância entre acoplamentos $21 \mathrm{~cm}$; folga $1 \mathrm{~mm} ; \Omega=12 \mathrm{~Hz}$ : a) $F_{i}$ versus $F_{0}$; b) acelerações.

4.56 Respostas no tempo. Distância entre acoplamentos $21 \mathrm{~cm}$; folga $1 \mathrm{~mm} ; \Omega=12.25 \mathrm{~Hz}$ : a) $F_{i}$ versus $F_{0}$; b) acelerações.

4.57 Respostas no tempo. Distância entre acoplamentos $21 \mathrm{~cm}$; folga $1 \mathrm{~mm} ; \Omega=13.75 \mathrm{~Hz}$ : a) $F_{i}$ versus $F_{0}$; b) acelerações.

4.58 Resposta no domínio da freqüência. Distância entre acoplamentos $21 \mathrm{~cm}$; folga $1 \mathrm{~mm} ; F_{i} / F_{0}$ versus $\Omega$.

4.59 Respostas no tempo. Distância entre acoplamentos $21 \mathrm{~cm}$; folga $3 \mathrm{~mm} ; \Omega=4.25 \mathrm{~Hz}:$ a) $F_{i}$ versus $F_{0}$; b) acelerações.

4.60 Respostas no tempo. Distância entre acoplamentos $21 \mathrm{~cm}$; folga $3 \mathrm{~mm} ; \Omega=12.25 \mathrm{~Hz}$ : a) $F_{i}$ versus $F_{0}$; b) acelerações.

4.61 Resposta no domínio da freqüência. Distância entre acoplamentos $21 \mathrm{~cm}$; folga $3 \mathrm{~mm} ; F_{i} / F_{0}$ versus $\Omega$.

4.62 Resposta no domínio da freqüência. Distância entre acoplamentos $21 \mathrm{~cm}$; comparação entre folgas. $F_{i} / F_{0}$ versus $\Omega$.

4.63 Otimização da força de impacto. Primeiro modo de vibração. $F_{i} / F_{0}$ versus $\Omega$.

4.64 Otimização da força de impacto. Primeiro modo de vibração. $F_{i} / F_{0}$ versus $\Omega$.

5.1 Força de impacto no tempo. a) Condição inicial de $7 \mathrm{~mm}$. b) condição inicial de $5 \mathrm{~mm}$.

5.2 Identificação dos parâmetros do impacto. Condição inicial de $7 \mathrm{~mm} .123$ 
5.3 Identificação dos parâmetros do impacto. Condição inicial de $5 \mathrm{~mm} .123$

5.4 Resultados numéricos; $F_{i}$ versus $\Omega$ : a) distância entre acoplamentos $15 \mathrm{~cm}$; b) distância entre acoplamentos $17 \mathrm{~cm}$.

5.5 Resultados numéricos; $F_{i}$ versus $\Omega$ : a) distância entre acoplamentos $19 \mathrm{~cm}$; b) distância entre acoplamentos $21 \mathrm{~cm}$.

5.6 Resultados numéricos; $F_{i}$ versus $\Omega$ : a) distância entre acoplamentos $19 \mathrm{~cm}$ (detalhe - primeiro modo); b) distância entre acoplamentos $17 \mathrm{~cm}$ (detalhe - segundo modo).

5.7 Comparação numérico-experimental; distância entre acoplamentos $15 \mathrm{~cm}$; gap $0 \mathrm{~mm} ; F_{i} / F_{0}$ versus $\Omega$.

5.8 Comparação numérico-experimental; distância entre acoplamentos $15 \mathrm{~cm}$; gap $1 \mathrm{~mm} ; F_{i} / F_{0}$ versus $\Omega$. 127

5.9 Comparação numérico-experimental; distância entre acoplamentos $15 \mathrm{~cm}$; gap $3 \mathrm{~mm} ; F_{i} / F_{0}$ versus $\Omega$. 128

5.10 Comparação numérico-experimental; distância entre acoplamentos $17 \mathrm{~cm}$; gap $0 \mathrm{~mm} ; F_{i} / F_{0}$ versus $\Omega$. 130

5.11 Comparação numérico-experimental; distância entre acoplamentos $17 \mathrm{~cm}$; gap $1 \mathrm{~mm} ; F_{i} / F_{0}$ versus $\Omega$. 130

5.12 Comparação numérico-experimental; distância entre acoplamentos $17 \mathrm{~cm}$; gap $3 \mathrm{~mm} ; F_{i} / F_{0}$ versus $\Omega$. 131

5.13 Comparação numérico-experimental; distância entre acoplamentos $19 \mathrm{~cm}$; gap $0 \mathrm{~mm} ; F_{i} / F_{0}$ versus $\Omega$. 132

5.14 Comparação numérico-experimental; distância entre acoplamentos $19 \mathrm{~cm} ;$ gap $1 \mathrm{~mm} ; F_{i} / F_{0}$ versus $\Omega$. 133

5.15 Comparação numérico-experimental; distância entre acoplamentos $19 \mathrm{~cm}$; gap $3 \mathrm{~mm} ; F_{i} / F_{0}$ versus $\Omega$. 133

5.16 Comparação numérico-experimental; distância entre acoplamentos $21 \mathrm{~cm}$; gap $0 \mathrm{~mm} ; F_{i} / F_{0}$ versus $\Omega$. 135

5.17 Comparação numérico-experimental; distância entre acoplamentos $21 \mathrm{~cm}$; gap $1 \mathrm{~mm} ; F_{i} / F_{0}$ versus $\Omega$. 135

5.18 Comparação numérico-experimental; distância entre acoplamentos $21 \mathrm{~cm}$; gap 3mm; $F_{i} / F_{0}$ versus $\Omega$. 136 


\section{Lista de Tabelas}

2.1 Parâmetros da simulação numérica. 42

3.1 Parâmetros: modelos de comparação. 57

3.2 Parâmetros do RIMD. 58

4.1 Especificação dos sensores. $\quad 72$

4.2 Especificação do shaker. 72

4.3 Massas dos componentes. $\quad 76$

4.4 Freqüências naturais, distância entre acoplamentos $10 \mathrm{~cm}$. $\quad 80$

4.5 Freqüências naturais, distância entre acoplamentos $12 \mathrm{~cm}$. $\quad 81$

4.6 Identificação de parâmetros - distância entre acoplamentos $15 \mathrm{~cm}$. 82

4.7 Freqüências naturais - comparação numérico-experimental, distância entre acoplamentos $15 \mathrm{~cm}$. 82

4.8 Freqüências naturais (experimentais) - sistema com e sem impacto, distância entre acoplamentos $15 \mathrm{~cm}$. 96

4.9 Identificação de parâmetros - Distância entre acoplamentos $17 \mathrm{~cm}$. 96

4.10 Freqüências naturais - comparação numérico-experimental, distância entre acoplamentos $17 \mathrm{~cm}$. 97

4.11 Freqüências naturais (experimentais) - sistema com e sem impacto, distância entre acoplamentos $17 \mathrm{~cm}$. 98

4.12 Identificação de parâmetros - Distância entre acoplamentos $19 \mathrm{~cm}$. 98

4.13 Freqüências naturais - comparação numérico-experimental, distância entre acoplamentos $19 \mathrm{~cm}$. 98

4.14 Freqüências naturais (experimentais) - sistema com e sem impacto, distância entre acoplamentos $19 \mathrm{~cm}$. 102

4.15 Identificação de parâmetros - Distância entre acoplamentos 21cm. 102

4.16 Freqüências naturais (experimentais) - sistema com e sem impacto, distância entre acoplamentos $21 \mathrm{~cm}$. 116

5.1 Parâmetros do RIMD para identificação dos parâmetros de $\begin{array}{ll} & 122 \\ \text { impacto. } & 122\end{array}$

5.2 Parâmetros do impacto. 122

5.3 Freqüências naturais; numérico e experimental; distância entre acoplamentos $15 \mathrm{~cm}$. 129

5.4 Forças de impacto máximas; numérico e experimental; distância entre acoplamentos $15 \mathrm{~cm}$.

5.5 Freqüências naturais; numérico e experimental; distância entre acoplamentos $17 \mathrm{~cm}$. 131

5.6 Forças de impacto máximas; numérico e experimental; distância entre acoplamentos $17 \mathrm{~cm}$.

5.7 Freqüências naturais; numérico e experimental; distância entre acoplamentos $19 \mathrm{~cm}$.

5.8 Forças de impacto máximas; numérico e experimental; distância entre acoplamentos $19 \mathrm{~cm}$. 
5.9 Freqüências naturais; numérico e experimental; distância entre acoplamentos $21 \mathrm{~cm}$. 136

5.10 Forças de impacto máximas; numérico e experimental; distância entre acoplamentos $21 \mathrm{~cm}$. 


\section{Lista de simbolos}

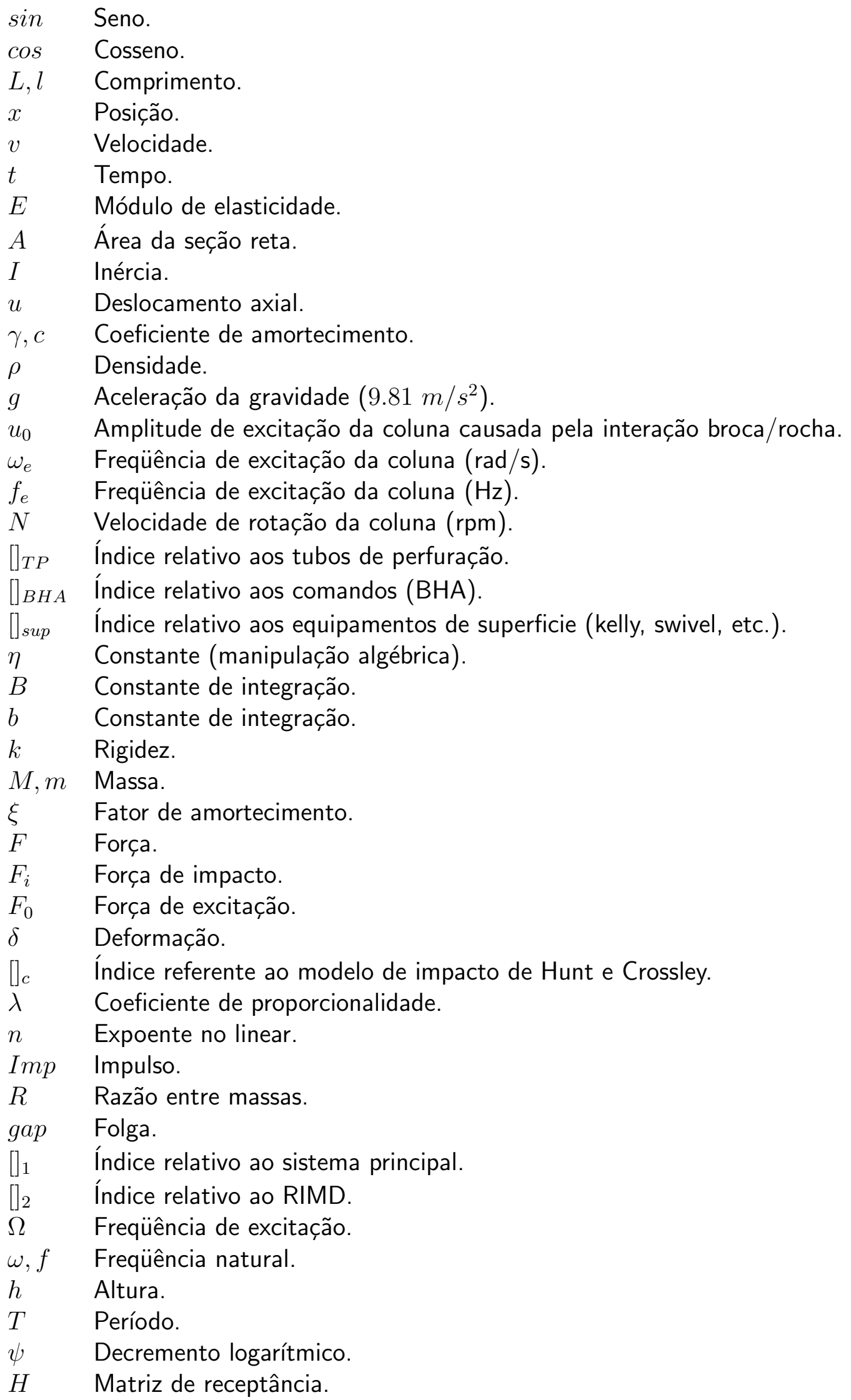


Uma jornada de mil milhas deve começar com um único passo.

Lao Tsé. 